\title{
Segment-specific association of carotid- intima-media thickness with cardiovascular risk factors - findings from the STAAB cohort study
}

\author{
Lara Müller-Scholden ${ }^{1}$ (D) Jan Kirchhof ${ }^{1}$, Caroline Morbach ${ }^{1,2}$, Margret Breunig ${ }^{1,2}$, Rudy Meijer ${ }^{3}$, Viktoria Rücker ${ }^{4}$, \\ Theresa Tiffe ${ }^{1,4}$, Tino Yurdadogan ${ }^{1}$, Martin Wagner ${ }^{4}$, Götz Gelbrich ${ }^{4,5}$, Michiel L. Bots ${ }^{3}$, Stefan Störk ${ }^{1,2^{*}+}$ and \\ Peter U. Heuschmann ${ }^{1,4,5+}$
}

\begin{abstract}
Background: The guideline recommendation to not measure carotid intima-media thickness (CIMT) for cardiovascular risk prediction is based on the assessment of just one single carotid segment. We evaluated whether there is a segment-specific association between different measurement locations of CIMT and cardiovascular risk factors.

Methods: Subjects from the population-based STAAB cohort study comprising subjects aged 30 to 79 years of the general population from Würzburg, Germany, were investigated. CIMT was measured on the far wall of both sides in three different predefined locations: common carotid artery (CCA), bulb, and internal carotid artery (ICA). Diabetes, dyslipidemia, hypertension, smoking, and obesity were considered as risk factors. In multivariable logistic regression analysis, odds ratios of risk factors per location were estimated for the endpoint of individual age- and sex-adjusted 75th percentile of CIMT.
\end{abstract}

Results: 2492 subjects were included in the analysis. Segment-specific CIMT was highest in the bulb, followed by CCA, and lowest in the ICA. Dyslipidemia, hypertension, and smoking were associated with CIMT, but not diabetes and obesity. We observed no relevant segment-specific association between the three different locations and risk factors, except for a possible interaction between smoking and ICA.

Conclusions: As no segment-specific association between cardiovascular risk factors and CIMT became evident, one simple measurement of one location may suffice to assess the cardiovascular risk of an individual.

Keywords: Carotid intima-media thickness (CIMT), Cardiovascular risk prediction, Carotid segment, Carotid ultrasound, Cardiovascular risk factors

\section{Background}

Many cardiovascular (CV) events occur in thitherto asymptomatic patients [1]. Therefore, it is important to improve assessment of subclinical vascular disease especially in subjects with an intermediate risk according to established risk prediction models [2, 3]. Measuring

\footnotetext{
* Correspondence: Stoerk_S@ukw.de

${ }^{+}$Störk Stefan and U. Heuschmann Peter contributed equally to this work. ${ }^{1}$ Comprehensive Heart Failure Center Würzburg, University and University Hospital Würzburg, Straubmühlweg 15, 97080 Würzburg, Germany

2Department of Internal Medicine I, University Hospital Würzburg, Würzburg, Germany

Full list of author information is available at the end of the article
}

carotid intima-media thickness (CIMT) via B-mode ultrasound presents a widely accepted, noninvasive, sensitive and reproducible technique to quantify subclinical vascular disease $[2,4,5]$. It is accepted that CIMT at three different locations, i.e. common carotid artery (CCA), carotid bulb, and internal carotid artery (ICA), is associated with established CV risk factors [6]. CIMT is also a generally acknowledged independent predictor for the occurrence of $\mathrm{CV}$ disease [7, 8]. Furthermore, interventional studies have shown that CIMT progression can be positively affected by appropriate treatment of $\mathrm{CV}$ risk factors [9]. However, current guidelines do not

(c) The Author(s). 2019 Open Access This article is distributed under the terms of the Creative Commons Attribution 4.0 International License (http://creativecommons.org/licenses/by/4.0/), which permits unrestricted use, distribution, and reproduction in any medium, provided you give appropriate credit to the original author(s) and the source, provide a link to the Creative Commons license, and indicate if changes were made. The Creative Commons Public Domain Dedication waiver (http://creativecommons.org/publicdomain/zero/1.0/) applies to the data made available in this article, unless otherwise stated. 
recommend the use of CIMT for systematic risk assessment in clinical practice for primary prevention $[10,11]$. This advice was based on meta-analyses failing to detect a clinically relevant improvement in the performance of common prediction models by adding CIMT $[12,13]$. Of note, these meta-analyses solely relied on CIMT of the CCA, and measurements of the bulb and/or ICA were not taken into consideration [14]. Further, these guidelines are controversially discussed as plaque prevalence, known as another important risk modifier, differs among sites [14]. Finally, the association between CIMT and risk factors as systolic blood pressure, cholesterol levels or smoking, seems to show a segment-specific effect $[6,15,16]$. Most of the other studies analyzing the segment-specific effect of different $\mathrm{CV}$ risk factors on CIMT originated from US populations $[6,15,16]$. These results are not readily generalizable to European populations because of different ethnicities and lifestyles.

Thus, the aim of this study was to examine whether there is a segment-specific association between the traditional CV risk factors and CIMT measured at different locations of the carotid artery in a representative sample from a German population. Furthermore, we aimed to assess, which segment might best reflect the impact of CV risk factors.

\section{Methods}

\section{Subjects}

The study sample derived from the population-based STAAB (Characteristics and Course of Heart Failure Stages A-B and Determinants of Progression) cohort study. The study design has been reported in detail previously [17]. Briefly, STAAB includes 5000 people to assess the prevalence of heart failure stages $\mathrm{A}$ and $\mathrm{B}$, and to investigate the progression from asymptomatic cardiac dysfunction into symptomatic heart failure [17]. Subjects were drawn randomly from a sample of the general population of the city of Würzburg. Subjects had to be aged between 30 and 79 years at the day of sampling. The only exclusion criterion was a pre-existing diagnosis of symptomatic heart failure. Baseline assessment took place from December 2013 to October 2017. All study procedures follow a priori defined measurement protocols are subjected to rigid quality control.

\section{Risk factor assessment}

Five risk factors were considered and the following definition were used. Manifest diabetes mellitus was assumed if the level of the HbA1c was above $6.5 \%$, or fasting plasma glucose level was above $7 \mathrm{mmol} / \mathrm{l}$, or if the subject was on anti-diabetic medication. Dyslipidemia was defined as total blood cholesterol level above $200 \mathrm{mg} / \mathrm{dl}$ after a fasting period of at least $10 \mathrm{~h}$, or use of lipid lowering drugs. Arterial hypertension was defined as blood pressure above $140 / 90 \mathrm{mmHg}$ or use of anti-hypertensive medication. All participants, who were current smokers or ex-smokers (defined as having smoked at least 100 cigarettes in their lifetime) at the time of examination, were counted as smokers. Smoking of cigars or pipe was also considered. Subjects were categorized as obese with a body mass index (BMI) above $30 \mathrm{~kg} / \mathrm{m}^{2}$. Blood pressure was the median of two to three measurements taken five minute apart in sitting position. Definitions of risk factors were used in previous publications from this study population [17, 18]. A subcohort of apparently healthy people free of any CV risk factor and without previous stroke or $\mathrm{CV}$ disease was also defined, and age- and sex-adjusted reference values of CIMT were generated for the population. In accordance with current guidelines, the 75th percentile was considered as cut-off-point indicating an increased individual risk for the manifestation of CV disesease [2].

\section{Carotid ultrasound}

All examiners performing sonographic measurements of subjects underwent an extended training and certification process conducted by an independent expert (RM) prior to the first examination. The training protocol included acquisition, storage, and analysis of the data following published standards [2]. Two different ultrasound devices were used to image the arteries (Vivid S and Vivid Q, General Electric Healthcare). CIMT was assessed via B-mode sonography using a 10 (Vivid Q) or $13 \mathrm{MHz}$ (Vivid S6) linear transducer (8 L-RS). Before the exact measurement of CIMT, a standardized screening of the carotid artery was performed, and the existence and distribution of plaques were captured. The far wall of three different, well-defined locations at both sides of the neck was measured during the end of diastole. The CCA was defined as the segment $10 \mathrm{~mm}$ prior to the beginning of the bifurcation. The bulb was defined as the segment from the beginning to the tip of the flow divider and ICA as the segment $10 \mathrm{~mm}$ after the tip of the flow divider. Pulsed wave Doppler and color Doppler were used to distinguish the internal from the external carotid artery. Each segment image was captured at the highest visible IMT using the ECG R-wave (end diastole). Plaques were defined as CIMT $>1.5 \mathrm{~mm}$ in one or more of the measured CIMT segments, and were included in the measurement. Images were stored and analyzed off-line using the Syngo Arterial Health Package (Syngo US Workplace, Siemens Medical Solutions USA, Inc.). A composite CIMT value was calculated as the mean of all available values (one to six). Reference values were generated for every location and the composite value. The average intraclass correlation coefficient (ICC) for interobserver reproducibility based on 40 double measurements was 0.78 (95\% CI: 
0.62-0.87) for composite CIMT, 0.67 (95\% CI: $0.48-$ 0.82 ) for right CCA, 0.63 (95\% CI: 0.36-0.80) for right bulb, 0.65 (95\% CI: 0.42-0.80) for right ICA, 0.65 (95\% CI: 0.42-0.80) for left CCA, 0.63 (95\% CI: 0.37-0.80) for left bulb, and 0.31 (95\% CI: 0.01-0.57) for left ICA, respectively.

\section{Statistical analysis}

For the comparison of continuous variables, t-test for independent samples after checking Levene's test for equality of variances was used. The chi-square test was used for the comparison of categorical variables. Multivariable logistic regression analysis was conducted using an inclusion-model considering diabetes, dyslipidemia, hypertension, smoking and obesity all in the same model. As outcome variable, the age- and sex-adjusted normative value above the 75th percentile was used for each location. A sensitivity analysis excluding apparently healthy subjects did not result in material changes of the estimates. Thus, the data of all subjects were included in the final analysis. Collinearity of $\mathrm{CV}$ risk factors was tested by computing the variance inflation factors (cut off value: 2). The age spectrum was divided into three equal age groups. Interactions between the different locations, the different age groups and the different risk factors were analyzed using generalized estimating equations (GEE). Logarithms of IMT values were computed to ensure standard distribution and used as dependent factor. For each risk factor a distinct age- and sex-adjusted model was calculated. All statistical analyses were performed using SPSS (version 24). P-values $<0.05$ were considered statistically significant.

\section{Results}

Between December 12, 2013 and March 1, 2016, the first 2492 people were examined. This number corresponds to the first 19 batches of the recruitment process. No CIMT values were available for $7 \%$ of the study sample. Distribution of missing values in the respective distinct location did not show a statistically significant association with the different locations or sides (right side: CCA $9 \%$, bulb $10 \%$, ICA $10 \%$; left side: CCA $9 \%$, bulb 9\%, ICA 10\%; all $p>0.05$ ). The general characteristics of the population are shown in Table 1 . A total of $76 \%$ of the whole population had at least one risk factor.

The composite CIMT was $0.68 \pm 0.17 \mathrm{~mm}$ for the overall population. Men had higher CIMT values than women in every location. CIMT was highest at the bulb, followed by CCA, and lowest at the ICA. As there were no differences between the IMT values of both sides of the neck $(p>0.05)$, mean values of both sides were calculated and used as independent variables for the following analysis. People with previous stroke, CV event or manifest peripheral arterial disease had higher CIMT
Table 1 Characteristics of the study population

\begin{tabular}{ll}
\hline Variable & Total $n=2492$ \\
\hline Men, $n$ (\%) & $1212(49)$ \\
Age [years], mean (SD) & $54 \pm 12$ \\
Agegroups $n$ (\%) & \\
$\quad<=45$ years & $597(24)$ \\
$\quad 46-60$ years & $1021(41)$ \\
$\quad>60$ years & $868(35)$ \\
Height [cm], mean (SD) & $171 \pm 9$ \\
Weight [kg], mean (SD) & $78 \pm 17$ \\
Obesity [BMl > 30 kg/m], $n$ (\%) & $492(20)$ \\
Blood pressure systolic [mmHg], mean (SD) & $131 \pm 18$ \\
Blood pressure diastolic [mmHg], mean (SD) & $79 \pm 10$ \\
Hypertension, $n$ (\%) & $879(38)$ \\
Total cholesterol [mg/dl], mean (SD) & $208 \pm 38$ \\
Dyslipidemia, $n$ (\%) & $338(15)$ \\
HbA1c [\%], mean (SD) & $5.5 \pm 0.61$ \\
Diabetes, $n$ (\%) & $217(9)$ \\
Current or ex-smoker, $n$ (\%) & $1324(54)$ \\
Current smoker, $n$ (\%) & $455(19)$ \\
Ex-smoker, $n$ (\%) & $869(35)$ \\
Previous cardiovascular disease, $n$ (\%) & $102(4)$ \\
Previous stroke, $n$ (\%) & $49(2)$ \\
\hline Apparently healthy, $n$ (\%) & $538(24)$ \\
\hline
\end{tabular}

values in any considered location (all $p<0.001$ ). Plaque prevalence was relatively low with a total number of 586 people $(26 \%)$ having at least one plaque in the investigated carotid artery segment (Table 2).

Multivariable logistic regression analysis showed that arterial hypertension, dyslipidemia and smoking were independent predictors for CIMT values above the ageand sex-adjusted 75th percentile (Table 3). While hypertension showed a significant effect on all locations measured, dyslipidemia demonstrated a statistically significant effect only at the bulb and ICA. In addition, a strong positive trend for the association of dyslipidemia with CCA was found. The effects of smoking were visible at the bulb and CCA. Diabetes mellitus and obesity seemed to exert no effects on any segments. Odds ratios for significant risk factors varied between 1.30 (for smoking and $\mathrm{CCA}$ ) and 1.86 (for hypertension and composite CIMT). No collinearity between the different $\mathrm{CV}$ risk factors was apparent. In GEE we found a significant interaction between the segment of measurement and the following risk factors: dyslipidemia $(p<0.001)$, hypertension $(p<0.001)$, and smoking $(p=0.002)$. No interaction was found between age in tertiles and the five major risk factors (all $p>0.05$ ). 
Table 2 Segment-specific assessment of CIMT

\begin{tabular}{ll}
\hline Variable & Total $\mathrm{n}=2492$ \\
\hline Mean RCCA [mm], mean (SD) & $0.66 \pm 0.17$ \\
Men [mm], mean (SD) & $0.68 \pm 0.18$ \\
Women [mm], mean (SD) & $0.64 \pm 0.15$ \\
Mean right bulb [mm], mean (SD) & $0.82 \pm 0.34$ \\
Men [mm], mean (SD) & $0.88 \pm 0.37$ \\
Women [mm], mean (SD) & $0.78 \pm 0.31$ \\
Mean RICA [mm], mean (SD) & $0.58 \pm 0.22$ \\
Men [mm], mean (SD) & $0.61 \pm 0.24$ \\
Women [mm], mean (SD) & $0.55 \pm 0.20$ \\
Mean left CCA [mm], mean (SD) & $0.66 \pm 0.18$ \\
Men [mm], mean (SD) & $0.68 \pm 0.18$ \\
Women [mm], mean (SD) & $0.63 \pm 0.16$ \\
Mean left bulb [mm], mean (SD) & $0.82 \pm 0.32$ \\
Men [mm], mean (SD) & $0.87 \pm 0.35$ \\
Women [mm], mean (SD) & $0.76 \pm 0.27$ \\
Mean left ICA [mm], mean (SD) & $0.57 \pm 0.21$ \\
Men, [mm], mean (SD) & $0.61 \pm 0.23$ \\
Women, [mm], mean (SD) & $0.54 \pm 0.17$ \\
Mean of both sides & \\
CCA [mm], mean (SD) & $0.66 \pm 0.15$ \\
Bulb [mm], mean (SD) & $0.82 \pm 0.28$ \\
ICA [mm], mean (SD) & $0.57 \pm 0.18$ \\
ICA & $5.68 \pm 0.17$ \\
\hline
\end{tabular}

\section{Discussion}

We analyzed the association between $\mathrm{CV}$ risk factors and different locations of CIMT in a population-based study from the general population of Würzburg, Germany. Our study comprised not only a broad age range but also a detailed assessment of $\mathrm{CV}$ risk profile and CIMT. Hypertension and dyslipidemia showed a significant association on having CIMT above the 75th percentile independent from the measurement location, while smoking seemed to have an effect on CCA and bulb only. Our results imply that no segment carries more information about the influence of $\mathrm{CV}$ risk factors on CIMT than the others.

In the STAAB cohort the prevalence of plaque was relatively low, with $26 \%$ of all people having at least one plaque in their carotid arteries. Other studies published plaque prevalences between 34 and $87 \%$ [19-21]. This prompts the question as to whether the plaque prevalence and lower CIMT is attributable to the reported population or if the methods of plaque acquisition differ $[20,22]$. One possible explanation for a lower prevalence of plaques observed here might be variations in uptake of preventive medication such as lipid-lowering drugs [18]. CIMT values were higher in men than women in all different locations with the highest CIMT values found in the bulb $[6,23]$. Other population studies reported the lowest values in the CCA, whereas in our study the lowest values were found in the ICA [23, 24]. As plaque prevalence in ICA is usually higher than in the CCA and plaque prevalence in the STAAB cohort is comparatively low this could explane this difference [25]. In line with previous studies, there was no difference between CIMT of right and left carotid arteries [26].

ICC for left internal carotid artery was remarkably low compared to the other segments $(0.31$ vs. 0.63 (right bulb) - 0.78 (composite CIMT)). Nichols et al. [27] report the best matches for the lateral images of the right side, therefore the handiness of the examiner could play a role since the right IMT is examined with the right hand and the left with the left and all examiners were right-handed. Regarding the particularly low ICC of the left internal carotid artery, this might be attributable to the fact that this segment is the most challenging for a right handed examiner.

Taking all CV risk factors in consideration, only diabetes and obesity seemed to have no association with CIMT. With regard to obesity, many studies also found no independent relation with CIMT as well $[6,28]$. Apparently, the known higher CV risk of obese people cannot be simply read off the CIMT [10]. BMI is believed to have no direct influence on CIMT, but rather

Table 3 Strength of the association between CV risk factor and CIMT value above the 75th percentile

\begin{tabular}{lllll}
\hline Risk factor & Composite CIMT & CCA & Bulb & ICA \\
\hline Diabetes & $1.24(0.89-1.71)$ & $1.22(0.87-1.70)$ & $1.10(0.78-1.54)$ & $1.29(0.91-1.80)$ \\
Dyslipidemia & $1.73(1.34-2.23)$ & $1.28(0.98-1.65)$ & $1.79(1.38-2.32)$ & $1.43(1.10-1.86)$ \\
Hypertension & $1.86(1.53-2.27)$ & $1.66(1.35-2.03)$ & $1.75(1.44-2.13)$ & $1.45(1.22-1.84)$ \\
Obesity & $0.91(0.72-1.16)$ & $0.95(0.74-1.21)$ & $0.88(0.69-1.12)$ & $1.25(0.98-1.60)$ \\
Smoking & $1.47(1.23-1.77)$ & $1.30(1.08-1.57)$ & $1.44(1.20-1.72)$ & $1.01(0.84-1.22)$ \\
\hline
\end{tabular}

Data are odds ratio (OR) with $95 \%$ confidence interval (CI) derived from multivariable regression analysis for the likelihood of having CIMT values above the 75th percentile 
may effect CIMT through other risk factors [28]. Further, we were unable to show a statistically significant effect of diabetes on CIMT. The pathophysiological concept renders glycated molecules in the arteries responsible for the observed greater $\mathrm{CV}$ risk of diabetic people, but in epidemiological studies this association is infrequently present $[29,30]$. The reasons for this finding are unclear and might be caused by the low number of cases or by collinearity of different factors that in multivariable analysis masking true effects $[29,30]$. In the present study 217 people (9\%) did present overt manifest diabetes mellitus. Thus, we consider the number of cases in the different locations as sufficient to detect an effect. In our population, diabetes did not contribute to CIMT thickness in a clinically relevant way, even if nearly $30 \%$ of people with an $\mathrm{HbA1c}>6.5 \%$ were diagnostically naïve and $44 \%$ of people with antidiabetic medication in the STAAB population were insufficiently treated [18].

Our findings confirm the importance of the three other risk factors dyslipidemia, hypertension and smoking that showed a significant effect on CIMT [6, 22, 30, 31]. Results of the logistic regression indicate that there was no segment-specific effect of hypertension and dyslipidemia. Only smoking showed a potential interaction with the ICA segment. However, previous studies reported a segment-specific effect and only disagreed on the segment which carries the most information $[6,15,16]$. Polak et al. considered the CCA as the location containing the most information, while Urbina et al. identified the bulb as the most informative segment $[15,16]$. There are only a few publications that also did not show a segment-specific influence of the traditional risk factors [26, 32, 33]. Variations between prvious studies might be caused by differences in analytical methodology. In contrast to previous publications we used different statistical methods. Rather than employing linear regression and comparing the magnitude of $R^{2}$ [2], we used logistic regression with the endpoint of CIMT $>75$ th percentile of ageand sex-adjusted reference values $[6,15]$. The most important advantage of this approach is that it portrays the clinically relevant effect of a $\mathrm{CV}$ risk factor. However, these different approaches render a direct comparison of our results with previous studies problematic. Using a linear analysis to detect possible interactions we did also find significant interactions between the location of measurement and the risk factors dyslipidemia, hypertension and smoking. Nevertheless, Lorenz et al. reported very similar hazard rate ratios for the three different locations and the occurrence of a CV event and concluded, as well as del Sol et al., that there is no difference in the power of risk prediction between segments [26, 33].

\section{Limitations and strengths}

We observed a lower prevalence of plaques than reported by others. Reproducibility of image acquisition and reading is an important element built into the STAAB study design [17, 34]. All technicians underwent a dedicated training and certification procedure and followed a structured protocol. Further, when analyzing the reproducibility at different locations we did not find a segment-specific difference. Nevertheless, as five sonographers were capturing images and reading CIMT along the study, a certain degree of variation must be considered as a potential confounding factor. The subgroup of apparently healthy people, forming the basis for the reference values, compared favourably with similar studies that aimed to generate reference values. The 75th percentile of "normal" values was lower in our sample than in previous studies [20]. This may be partially due to the strict definition of the risk factor "smoker" that included all people ever smoking more than 100 cigarettes or cigars in their life - regardless of the current smoking status. This was based on previous studies reporting an increase in CIMT and progression rates up to 2 or 3 years after smoking cessation [35, 36]. However, this approach excludes people with a very low tobacco consumption, who may not have subclinical atherosclerosis caused by smoking. The size of our study sample provides for sufficient statistical power analyzing subgroups and comparisons with previous population-based studies $[15,29]$. However, the generalizability may be higher in groups of middle-aged people with very frequent $\mathrm{CV}$ risk factors like hypertension and smoking in contrast to other population groups. As we observed no significant interaction between age groups and risk factor level, we consider it unlikely that the variation in age may be a major confounder of our results.

\section{Conclusion}

The results of our study support the hypothesis that a CIMT measurement derived at a single segment provides sufficient information about the association between risk factors and CIMT. As there is no preference for a specific segment nor side for risk factors, a simple measurement executed at one location on either side of the neck might be sufficient to allow $\mathrm{CV}$ risk assessment in an individual. Further research should focus on the value of the segment-specific effects of plaques in risk prediction.

\footnotetext{
Abbreviations

CCA: Common carotid artery; CIMT: Carotid intima-media-thickness: CV: Cardiovascular; GEE: Generalized estimating equations; ICA: Internal carotid artery; ICC: Intraclass correlation coefficient; STAAB: Characteristics and course of heart failure stages $A-B$ and determinants of progression
} 


\section{Acknowledgements}

We greatly appreciate the time of all STAAB participants and their willingness to provide data to the study. We also thank the Mayor of the City of Würzburg and the local registration office for their kind and sustained support of our study. Finally, we thank the entire study team including $\mathrm{K}$. Haas, M. Oezkur, M. Reuter, J. Kircher, T. Ludwig, study nurses, technicians, data managers, and students for their efforts on the STAAB study. We also thank M. Ertl, G. Fette, F. Puppe from the CHFC DataWarehouse, Institute of Informatics VI, University of Würzburg [18].

\section{Funding}

The STAAB project is a joint initiative of the Comprehensive Heart Failure Centre Würzburg (CHFC) and the Institute of Clinical Epidemiology and Biometry, Würzburg. The CHFC is funded by the German Ministry of Education and Research (BMBF 01EO1004 and 01EO1504). LMS received a scholarship from the Graduate School of Life Sciences (GSLS) of the University of Würzburg from 2015 to 2016. The GSLS supports doctoral students financially in order to devote a whole semester entirely to research - independent of the project. MLB is scientific director of the Vascular Imaging Center, an international core laboratory for CIMT studies. This publication was funded by the German Research Foundation (DFG) and the University of Wuerzburg in the funding programme Open Access Publishing.

\section{Availability of data and materials}

The datasets used and/or analysed during the current study are available from the corresponding author on reasonable request.

\section{Authors' contributions}

LMS has contributed to conception and design, acquisition, analysis, interpretation and drafted the manuscript. JK has contributed to conception and acquisition. CM has contributed to conception, acquisition and interpretation. MB has contributed to conception, acquisition and interpretation. RM contributed to conception, design and interpretation. VR has contributed to conception, analysis and interpretation. TT has contributed to conception and design, acquisition and interpretation. TY has contributed to conception and acquisition. MW has contributed to conception and design, acquisition and interpretation. GG has contributed to conception, analysis and interpretation. MLB has contributed to conception, analysis and interpretation. SS has contributed to conception and design, acquisition and interpretation. PUH has contributed to conception and design, acquisition, analysis, interpretation and drafted the manuscript. All authors critically revised the manuscript, gave final approval and agree to be accountable for all aspects of work ensuring integrity and accuracy.

\section{Ethics approval and consent to participate}

The STAAB study was approved by the Ethics Committee of the Medical Faculty of the University Würzburg (\# 98/13). All participants provided written informed consent.

\section{Consent for publication}

Not applicable.

\section{Competing interests}

PUH reports grants from German Ministry of Research and Education, European Union, Charité-Universitätsmedizin Berlin, Berlin Chamber of Physicians, German Parkinson Society, University Hospital Würzburg, Robert-Koch-Institute, German Heart Foundation, Federal Joint Committee (G-BA) within the Innovationfond, University Hospital Heidelberg (within RASUNOA-prime; supported by an unrestricted research grant to the University Hospital Heidelberg from Bayer, BMS, Boehringer-Ingelheim, Daiichi Sankyo), Charité-Universitätsmedizin Berlin (within Mondafis; supported by an unrestricted research grant to the Charité from Bayer), University Göttingen (within FIND-AF randomized; supported by an unrestricted research grant to the University Göttingen from Boehringer-Ingelheim), outside the submitted work; The other authors declare that there is no conflict of interest.

\section{Publisher's Note}

Springer Nature remains neutral with regard to jurisdictional claims in published maps and institutional affiliations.

\section{Author details}

${ }^{1}$ Comprehensive Heart Failure Center Würzburg, University and University Hospital Würzburg, Straubmühlweg 15, 97080 Würzburg, Germany. ${ }^{2}$ Department of Internal Medicine I, University Hospital Würzburg, Würzburg, Germany. ${ }^{3}$ Julius Center for Health Sciences and Primary Care, University Medical Center Utrecht, Utrecht, The Netherlands. ${ }^{4}$ Institute of Clinical Epidemiology and Biometry, University of Würzburg, Würzburg, Germany. ${ }^{5}$ Clinical Trial Center, University Hospital Würzburg, Würzburg, Germany.

Received: 7 November 2018 Accepted: 14 March 2019 Published online: 04 April 2019

\section{References}

1. Michos ED, Nasir K, Braunstein JB, et al. Framingham risk equation underestimates subclinical atherosclerosis risk in asymptomatic women. Atherosclerosis. 2006;184:201-6. 2005/05/24. https://doi.org/10.1016/j. atherosclerosis.2005.04.004.

2. Stein JH, Korcarz CE, Hurst RT, et al. Use of carotid ultrasound to identify subclinical vascular disease and evaluate cardiovascular disease risk: a consensus statement from the American Society of Echocardiography Carotid Intima-Media Thickness Task Force. Endorsed by the Society for Vascular Medicine. J Am Soc Echocardiogr. 2008;21:93-111; quiz 189-190. 2008/02/12. https://doi.org/10.1016/j.echo.2007.11.011.

3. Touboul PJ, Hennerici MG, Meairs S, et al. Mannheim carotid intimamedia thickness and plaque consensus (2004-2006-2011). An update on behalf of the advisory board of the $3 \mathrm{rd}$, 4th and 5th watching the risk symposia, at the 13th, 15th and 20th European stroke conferences, Mannheim, Germany, 2004, Brussels, Belgium, 2006, and Hamburg, Germany, 2011. Cerebrovasc Dis. 2012;34:290-6. https://doi.org/10.1159/ 000343145.

4. Nichols S, Milner M, Meijer R, et al. Variability of automated carotid intimamedia thickness measurements by novice operators. Clin Physiol Funct Imaging. 2014 2014/09/13. https://doi.org/10.1111/cpf.12189.

5. Aldridge ME, Do K, Oo T, et al. Carotid intima-media thickness and plaque assessment by trained medical residents: validation and preliminary testing of a training protocol. J Am Soc Echocardiogr. 2013;26:1457-64. 2013/09/21. https://doi.org/10.1016/j.echo.2013.08.009.

6. Paul TK, Chen W, Srinivasan SR, et al. Contrast of the impact of multiple cardiovascular risk factors on the femoral and carotid intima-media thickness in asymptomatic young adults: the Bogalusa Heart Study. Atherosclerosis. 2011;216:359-64. 2011/03/08. https://doi.org/10.1016/j. atherosclerosis.2011.02.023.

7. Lorenz MW, Markus HS, Bots ML, et al. Prediction of clinical cardiovascular events with carotid intima-media thickness: a systematic review and metaanalysis. Circulation. 2007;115:459-67. https://doi.org/10.1161/ CIRCULATIONAHA.106.628875.

8. de Groot E, Hovingh GK, Wiegman A, et al. Measurement of arterial wall thickness as a surrogate marker for atherosclerosis. Circulation. 2004;109: III33-8. https://doi.org/10.1161/01.CIR.0000131516.65699.ba.

9. Crouse JR 3rd, Raichlen JS, Riley WA, et al. Effect of rosuvastatin on progression of carotid intima-media thickness in low-risk individuals with subclinical atherosclerosis: the METEOR Trial. JAMA. 2007;297:1344-53. 2007/ 03/27. https://doi.org/10.1001/jama.297.12.1344.

10. Piepoli MF, Hoes AW, Agewall S, et al. 2016 European Guidelines on cardiovascular disease prevention in clinical practice: The Sixth Joint Task Force of the European Society of Cardiology and Other Societies on Cardiovascular Disease Prevention in Clinical Practice (constituted by representatives of 10 societies and by invited experts) Developed with the special contribution of the European Association for Cardiovascular Prevention \& Rehabilitation (EACPR). Atherosclerosis. 2016;252:207-74. 2016/ 09/25. https://doi.org/10.1016/j.atherosclerosis.2016.05.037.

11. Goff DC Jr, Lloyd-Jones DM, Bennett G, et al. 2013 ACC/AHA guideline on the assessment of cardiovascular risk: a report of the American College of Cardiology/American Heart Association task force on practice guidelines. Circulation. 2014;129:S49-73. https://doi.org/10.1161/01.cir.0000437741. 48606.98 .

12. van den Oord SC, Sijbrands EJ, ten Kate GL, et al. Carotid intima-media thickness for cardiovascular risk assessment: systematic review and metaanalysis. Atherosclerosis. 2013;228:1-11. https://doi.org/10.1016/j. atherosclerosis.2013.01.025 
13. Den Ruijter HM, Peters SE, Anderson TJ, et al. Common carotid intimamedia thickness measurements in cardiovascular risk prediction: a metaanalysis. JAMA. 2012;308:796-803. https://doi.org/10.1001/jama.2012.9630.

14. Stein JH, Tattersall MC. Carotid intima-media thickness and cardiovascular disease risk prediction. J Am Coll Cardiol. 2014;63:2301-2. 2014/03/13. https://doi.org/10.1016/j.jacc.2014.02.528.

15. Polak JF, Person SD, Wei GS, et al. Segment-specific associations of carotid intima-media thickness with cardiovascular risk factors: the Coronary Artery Risk Development in Young Adults (CARDIA) study. Stroke. 2010;41:9-15. 2009/11/17. https://doi.org/10.1161/strokeaha.109.566596.

16. Urbina EM, Srinivasan SR, Tang R, et al. Impact of multiple coronary risk factors on the intima-media thickness of different segments of carotid artery in healthy young adults (The Bogalusa Heart Study). Am J Cardiol. 2002;90:953-8 2002/10/26.

17. Wagner M, Tiffe T, Morbach C, et al. Characteristics and Course of Heart Failure Stages A-B and Determinants of Progression - design and rationale of the STAAB cohort study. European J Preventive Cardiol. 2017;24:468-79. 2016/11/24. https://doi.org/10.1177/2047487316680693.

18. Tiffe $T$, Wagner $M$, Rucker $V$, et al. Control of cardiovascular risk factors and its determinants in the general population- findings from the STAAB cohort study. BMC Cardiovasc Disord. 2017;17:276. 2017/11/04. https://doi.org/10. 1186/s12872-017-0708-X.

19. Mackinnon AD, Jerrard-Dunne P, Porteous $L$, et al. Carotid intima-media thickness is greater but carotid plaque prevalence is lower in black compared with white subjects. AJNR Am J Neuroradiol. 2010;31:1951-5. 2010/09/25. https://doi.org/10.3174/ajnr.A2214.

20. von Sarnowski B, Ludemann J, Volzke H, et al. Common carotid intimamedia thickness and framingham risk score predict incident carotid atherosclerotic plaque formation: longitudinal results from the study of health in Pomerania. Stroke. 2010;41:2375-7. 2010/09/04. https://doi.org/10. 1161/strokeaha.110.593244.

21. Ihle-Hansen $\mathrm{H}$, Vigen $\mathrm{T}$, Ihle-Hansen $\mathrm{H}$, et al. Prevalence of Carotid Plaque in a 63- to 65-Year-Old Norwegian Cohort From the General Population: The ACE (Akershus Cardiac Examination) 1950 Study. J American Heart Assoc. 2018;7:2018/05/10. https://doi.org/10.1161/jaha.118.008562.

22. Sinning C, Wild PS, Echevarria FM, et al. Sex differences in early carotid atherosclerosis (from the community-based Gutenberg-Heart Study). Am J Cardiol. 2011;107:1841-7. 2011/04/13. https://doi.org/10.1016/j.amjcard.2011. 02.318.

23. Howard G, Sharrett AR, Heiss G, et al. Carotid artery intimal-medial thickness distribution in general populations as evaluated by B-mode ultrasound. ARIC Investigators. Stroke. 1993;24:1297-304 1993/09/01.

24. Stensland-Bugge E, Bonaa KH, Joakimsen O. Age and sex differences in the relationship between inherited and lifestyle risk factors and subclinical carotid atherosclerosis: the Tromso study, Atherosclerosis. 2001;154:437-48 2001/02/13

25. Joakimsen O, Bonaa KH, Stensland-Bugge E, et al. Age and sex differences in the distribution and ultrasound morphology of carotid atherosclerosis: the Tromso Study. Arterioscler Thromb Vasc Biol. 1999;19:3007-13 1999/12/11.

26. Lorenz MW, von Kegler S, Steinmetz H, et al. Carotid intima-media thickening indicates a higher vascular risk across a wide age range: prospective data from the carotid atherosclerosis progression study (CAPS). Stroke. 2006;37:87-92. https://doi.org/10.1161/01.STR.0000196964.24024.ea.

27. Nichols S, Milner M, Meijer R, et al. Variability of automated carotid intimamedia thickness measurements by novice operators. Clin Physiol Funct Imaging. 2016;36:25-32. 2014/09/13. https://doi.org/10.1111/cpf.12189.

28. Gao Z, Khoury PR, McCoy CE, et al. Adiposity has no direct effect on carotid intima-media thickness in adolescents and young adults: Use of structural equation modeling to elucidate indirect \& direct pathways. Atherosclerosis. 2016;246:29-35. 2016/01/12. https://doi.org/10.1016/j.atherosclerosis.2015.11.033.

29. Baldassarre D, Nyyssonen K, Rauramaa R, et al. Cross-sectional analysis of baseline data to identify the major determinants of carotid intima-media thickness in a European population: the IMPROVE study. European Heart J. 2010;31:614-22. 2009/12/03. https://doi.org/10.1093/eurheartj/ehp496.

30. Eikendal AL, Groenewegen KA, Anderson TJ, et al. Common carotid intimamedia thickness relates to cardiovascular events in adults aged $<45$ years. Hypertension. 2015;65:707-13. https://doi.org/10.1161/HYPERTENSIONAHA. 114.04658.

31. Bauer M, Delaney JA, Mohlenkamp S, et al. Comparison of factors associated with carotid intima-media thickness in the Multi-ethnic Study of Atherosclerosis (MESA) and the Heinz Nixdorf Recall Study (HNR). J Am Soc
Echocardiogr. 2013;26:667-73. 2013/04/25. https://doi.org/10.1016/j.echo. 2013.03.011.

32. Stensland-Bugge $\mathrm{E}$, Bonaa $\mathrm{KH}$, Joakimsen $\mathrm{O}$, et al. Sex differences in the relationship of risk factors to subclinical carotid atherosclerosis measured 15 years later : the Tromso study. Stroke. 2000;31:574-81 2000/03/04.

33. Iglesias del Sol A, Bots ML, Grobbee DE, et al. Carotid intima-media thickness at different sites: relation to incident myocardial infarction; The Rotterdam Study. European Heart J. 2002;23:934-40. 2002/06/19. https://doi.org/10.1053/euhj.2001.2965.

34. Morbach C, Gelbrich G, Breunig M, et al. Impact of acquisition and interpretation on total inter-observer variability in echocardiography: results from the quality assurance program of the STAAB cohort study. Int J Cardiovasc Imaging. 2018;34:1057-65. 2018/02/16. https://doi.org/10.1007/ s10554-018-1315-3.

35. van den Berkmortel FW, Wollersheim $\mathrm{H}$, van Langen $\mathrm{H}$, et al. Two years of smoking cessation does not reduce arterial wall thickness and stiffness. Neth J Med. 2004;62:235-41 2004/11/24.

36. Johnson HM, Piper ME, Baker TB, et al. Effects of smoking and cessation on subclinical arterial disease: a substudy of a randomized controlled trial. PLoS One. 2012;7:e35332. 2012/04/13. https://doi.org/10.1371/journal.pone. 0035332.

\section{Ready to submit your research? Choose BMC and benefit from:}

- fast, convenient online submission

- thorough peer review by experienced researchers in your field

- rapid publication on acceptance

- support for research data, including large and complex data types

- gold Open Access which fosters wider collaboration and increased citations

- maximum visibility for your research: over $100 \mathrm{M}$ website views per year

At BMC, research is always in progress.

Learn more biomedcentral.com/submissions 